\title{
TRACES ON IRREGULAR IDEALS
}

\author{
JÓZSEF V. VARGA \\ (Communicated by John B. Conway)
}

\begin{abstract}
Simple answers are given to the following and related questions: For what Hilbert space operator $A$ is it true that the smallest ideal (alternatively, the smallest norm ideal, the smallest maximal norm ideal) containing $A$ is a norm (an intermediate norm, a principal norm) ideal? Do these ideals support a nontrivial unitary invariant positive linear functional?
\end{abstract}

\section{INTRODUCTION}

Let $\mathscr{H}$ be an infinite dimensional separable complex Hilbert space. By operators we shall mean bounded linear transformations of $\mathscr{H}$ into itself. Their algebra is denoted by $\mathscr{B}(\mathscr{H})$, while $\mathscr{K}(\mathscr{H})$ is the algebra of all compact operators. By an ideal we mean a two-sided ideal in (unless otherwise stated) $\mathscr{B}(\mathscr{H})$.

Definition. We say that the function $f$ from the ideal $\mathscr{I}$ into $\mathbf{C}$ is a trace on $\mathscr{I}$ if

(i) $f$ is linear,

(ii) $f\left(U^{*} X U\right)=f(X)$ for $X \in \mathscr{I}$ and $U^{-1}=U^{*} \in \mathscr{B}(\mathscr{H})$ ( $f$ is unitary invariant),

(iii) $f(X) \geq 0$ if $X \geq 0(f$ is positive).

In other words a trace is a positive unitary invariant linear (p.u.l.) functional. We note that (ii) is equivalent to the usual requirement that $f(A X)=f(X A)$ for $A \in \mathscr{B}(\mathscr{H})$ and $X \in \mathscr{I}$, since every operator is a linear combination of unitary operators. Sometimes it is included in the definition of a trace that $X \geq 0$ and $f(X)=0$ implies $X=0$ ( $f$ is faithful) or even (instead of (iii)) that its value is 1 on every rank 1 projection. As usual, we denote this classical trace on the ideal $\mathscr{F}(\mathscr{H})$ of finite rank operators by "tr." If $A \in \mathscr{K}(\mathscr{H})$ we write $\mathscr{I}(A)$ for the principal ideal generated by $A$. Later we shall introduce $\mathscr{N}(A)$ as the principal norm ideal generated by $A$, i.e. the smallest norm ideal containing $A$ (see Lemma 5), and $\mathscr{M}(A)$ as the maximal norm ideal containing

Received by the editors March 1, 1989.

1980 Mathematics Subject Classification (1985 Revision). Primary 47D25.

Key words and phrases. Compact operator, principal ideal, unitary invariance, $s$-numbers. 
$\mathscr{N}(A)$ as a closed subspace. The results of this paper can be summarized in the following two theorems:

Theorem IRR. For a compact operator $A$, the following conditions are equivalent:

(a) $\mathscr{I}(A)$ supports a nontrivial trace.

(b) $\mathscr{N}(A)$ supports a nontrivial trace.

(c) $\mathscr{M}(A)$ supports a nontrivial trace.

(d) $\mathscr{I}(A)$ is not a norm ideal.

(e) $\mathscr{N}(A)$ is not a principal ideal.

(f) $A$ is irregular (see definition below).

Theorem ECC. For a compact operator $A$, the following conditions are equivalent:

(g) $\mathscr{N}(A)$ is an intermediate norm ideal (see Remark 5).

(h) $\mathscr{M}(A)$ is not a principal norm ideal.

(i) $\left(l^{\infty} / c_{0}\right)^{*} \hookrightarrow \mathscr{N}(A)^{* u}$ (the unitary invariant part of $\left.\mathscr{N}(A)^{*}\right)$ by a positive linear isometry.

(j) $\left(l^{\infty} / c_{0}\right)^{*} \hookrightarrow \mathscr{M}(A)^{* u}$ by a positive linear isometry.

(k) $A$ is eccentric (see definition below).

In particular, no principal ideal is a separable norm ideal [4] or an intermediate norm ideal. Notice that if $A$ is not in the trace class, then all the above conditions $(a), \ldots,(\mathbf{k})$ are equivalent. To avoid confusion we note that the class of principal norm ideals strictly contains the class of those principal ideals which are norm ideals (see Theorem IRR).

\section{PRINCIPAL IDEALS}

Which ideals support a nontrivial trace? We give the answer here for the class of principal ideals and certain norm ideals related to them. If $A \in \mathscr{K}(\mathscr{H})$, we write $\mathscr{I}(A)$ for the principal ideal generated by $A$. In general we denote by $a_{n}$ the $n$th member of a sequence $a,(n=1,2, \ldots)$. So, for example, if $A \in \mathscr{K}(\mathscr{H})$, then $s(A)$ is the sequence of $s$-numbers of $A$ [2]; that is $s_{n}(A)$ is the $n$th eigenvalue of the square root of $A^{*} A$ counted in a decreasing order according to multiplicites. We find it convenient to write $S$ for the sequence of partial sums of $s: S_{n}=\sum_{i=1}^{n} s_{i}$. We split $\mathscr{K}(\mathscr{H}) \backslash\{0\}$ into three disjoint classes (r., n., e.) by the following:

Definition. Let $s$ be a nonincreasing sequence of nonnegative numbers for which $s_{1}>0$. We say that $s$ is

(r.) regular if $S_{n}=O\left(n s_{n}\right) \quad(n \rightarrow \infty)$ [2],

(i.) irregular if it is not regular,

(n.) nuclear if $\sum s_{n}<\infty$,

(e.) eccentric if it is irregular but not nuclear. 
We call a nonzero operator $A \in \mathscr{K}(H)$ regular (irregular, nuclear, eccentric) if $s(A)$ is regular (irregular, nuclear, eccentric resp.).

Lemma 1. The following conditions are equivalent:

(i) $s$ is irregular,

(ii) $\inf _{n} S_{2 n} / S_{n}=1$,

(iii) $\inf _{n} S_{k n} / S_{n}=1$ for every positive integer $k$,

(iv) inf ${ }_{n} S_{k n} S_{n}<3$ for every positive integer $k$,

(v) $\lim _{k \rightarrow \infty} S_{3 k n_{k}} / S_{n_{k}}=1$ for some strictly increasing sequence $\left(n_{k}\right)$ of positive integers.

Proof. The equivalence of (i) and (ii) follows from the obvious relation

$$
1+\frac{n s_{2 n}}{S_{2 n}} \leq 1+\frac{n s_{2 n}}{S_{n}} \leq \frac{S_{2 n}}{S_{n}} \leq 1+\frac{n s_{n}}{S_{n}} .
$$

We leave the rest of the proof to the reader, hinting only that $S$ is a concave sequence.

Note that nuclearity implies irregularity and that $\left(n^{a}\right)_{n=1}^{\infty}$ is regular if $-1<$ $a<0$ but is eccentric if $a=-1$. Also, there are regular as well as eccentric sequences outside of $\bigcup_{p<\infty} l^{p}$. If $A, B \in \mathscr{K}(\mathscr{H}), X, Y \in \mathscr{B}(\mathscr{H}), n, m=$ $1,2, \ldots$, we have (see [2])

$$
\begin{gathered}
s_{n}(X A Y) \leq\|X\| s_{n}(A)\|Y\|, \\
s_{m+n-1}(A+B) \leq s_{m}(A)+s_{n}(B) .
\end{gathered}
$$

Let $B=\sum_{i=1}^{r} X_{i} A Y_{i},\left(X_{i}, Y_{i} \in \mathscr{B}(\mathscr{H}), i=1,2, \ldots, r\right)$. Then it follows that

$$
s_{r(n-1)+1}(B) \leq \sum_{i=1}^{r} s_{n}\left(X_{i} A Y_{i}\right) \leq s_{n}(A) \sum_{i=1}^{r}\left\|X_{i}\right\|\left\|Y_{i}\right\| .
$$

Denote by $\mathscr{I}_{r}(A)$ the set of all operators $B$ in the above form. We have $\mathscr{F}(A)=\bigcup_{r=1}^{\infty} \mathscr{I}_{r}(A)$. Clearly (1) proves half of the following:

Lemma 2. $B \in \mathscr{I}_{r}(A)$ if and only if

$$
s_{r(n-1)+1}(B) \leq K s_{n}(A)
$$

for every positive integer $n$ with some constant $K$.

For the "if" part we note that

$$
s_{r n}(B) \leq s_{r n-1}(B) \leq \cdots \leq s_{r(n-1)+1}(B) \leq K s_{n}(A) \quad(n=1,2, \ldots)
$$

so the construction of the $X_{i}$ 's and $Y_{i}$ 's is easy through partial isometries. We refer to [2] for the proof of the following lemma:

Lemma 3. If $A \in \mathscr{K}(\mathscr{H})$ and $P$ is an arbitrary orthogonal projection with rank $P \leq n$, then

$$
|\operatorname{tr}(A P)| \leq S_{n}(A P) \leq S_{n}(A)
$$


Theorem 1. If $A \in \mathscr{K}(\mathscr{H})$ then $\mathscr{I}(A)$ is the domain of a nonzero trace if and only if $A$ is irregular.

Proof. Fix $A$ and put $s=s(A)$ for brevity. Let $B$ be an arbitrary compact operator, let $e(B)$ be an orthonormal sequence in $\mathscr{H}$ such that $B^{*} B e_{n}=$ $s_{n}^{2}(B) e_{n}(B)$, and let $E_{n}(B)$ be the orthogonal projection onto the subspace $\mathscr{H}_{n}(B)$ spanned by $e_{1}(B), e_{2}(B), \ldots e_{n}(B) \quad(n=1,2, \ldots)$. Suppose $A$ is irregular. Fix $\left(n_{k}\right)$ as in (v) of Lemma 1 . For any sequence $P$ of orthogonal projections we define a complex sequence as follows:

$$
\phi_{P}(B)(k)=\frac{\operatorname{tr}\left(B P_{k}\right)}{S_{n_{k}}}, \quad(k=1,2, \ldots) .
$$

The sequence $\phi_{P}(B)$ is bounded if $B \in \mathscr{F}(A)$ and the rank of $P_{k}$ is at most $M k n_{k}$ where $M$ is any positive integer. Indeed, $B \in \mathscr{I}_{r}(A)$ for some $r$ and so

$$
\left|\operatorname{tr}\left(B P_{k}\right)\right| \leq S_{M k n_{k}}(B) \leq M S_{k n_{k}}(B) \leq r M S_{k n_{k}}
$$

by Lemma 3, by (2), and by the concavity of the sequence $S(A)$. Let $P_{k}(B)=$ $E_{k n_{k}}(B)$ and $\phi(B)=\phi_{P(B)}(B)$. Obviously $\phi$ is a positive unitary invariant nonlinear map from $\mathscr{I}(A)$ into $l^{\infty}$. If $\pi$ denotes the factor map from $l^{\infty}$ onto $l^{\infty} / c_{0}$ then put $\tau_{P}=\pi \circ \phi_{P}$ where it is defined and let $\tau=\pi \circ \phi \mid \mathcal{F}(A)$. It is clear that $\tau$ is unitary invariant and, since $\phi$ and $\pi$ are positive, so is $\tau$. We claim that $\tau$ is even linear.

Definition. For two sequences $P$ and $R$ of orthogonal projections, let us say that $R$ is tied to $P$ if $P_{k} \leq R_{k}$ and the rank of $R_{k}$ is at most three times the rank of $P_{k}(k=1,2, \ldots)$.

We need the following result:

Lemma 4. If $B \in \mathscr{I}(A)$ and $R$ is tied to $P(B)$, then $\tau_{R}(B)=\tau(B)$.

Proof. Let $r$ be such that $B \in \mathscr{I}_{r}(A)$ and suppose that $k \geq r$. Then

$$
\begin{aligned}
\mid \phi_{R}(B) & -\phi(B)\left|S_{n_{k}}=\right| \operatorname{tr}\left(B R_{k}\right)-\operatorname{tr}\left(B P_{k}(B)\right)|=| \operatorname{tr}\left(B\left(R_{k}-P_{k}(B)\right)\right) \mid \\
& \leq S_{2 k n_{k}}\left(B\left(R_{k}-P_{k}(B)\right)\right)=S_{3 k n_{k}}(B)-S_{k n_{k}}(B) \leq S_{3 r k n_{k}}(B)-S_{r n_{k}}(B) \\
& =\sum_{i=r n_{k}+1}^{3 r k n_{k}} s_{i}(B)=\sum_{j=n_{k}+1}^{3 k n_{k}} \sum_{l=1}^{r} s_{r(j-1)+l}(B) \leq \sum_{j=n_{k}+1}^{3 k n_{k}} r K s_{j}(A) \\
& =r K\left(S_{3 k n_{k}}-S_{n_{k}}\right)=o\left(S_{n_{k}}\right) \quad(k \rightarrow \infty)
\end{aligned}
$$

by Lemmas 3 and 2 and by (2). This proves Lemma 4.

Let $B_{1}$ and $B_{2}$ be two arbitrary elements of $\mathscr{I}(A)$ and put $B_{3}=B_{1}+B_{2}$. Let $R_{k}$ be the orthogonal projection onto the span of $\bigcup_{i=1}^{3} \mathscr{H}_{k n_{k}}\left(B_{i}\right) \quad(k=$ $1,2, \ldots)$. Then $R$ is tied to $P\left(B_{i}\right) \quad(i=1,2,3)$, so $\tau\left(B_{i}\right)=\tau_{R}\left(B_{i}\right) \quad(i=$ $1,2,3)$ by the above lemma. But $\phi_{R}$ is clearly linear and so is $\tau_{R}$. Consequently $\tau\left(B_{3}\right)=\tau_{R}\left(B_{3}\right)=\tau_{R}\left(B_{1}\right)+\tau_{R}\left(B_{2}\right)=\tau\left(B_{1}\right)+\tau\left(B_{2}\right)$, so $\tau$ is additive. 
It is also homogeneous by the obvious homogeneity of $\phi$. So $\tau$ is a p.u.l. map, which proves our claim. Now, to conclude the proof of the "if" part we note that $\tau(|A|)=\pi(1,1,1, \ldots)$. Let $p$ be any nontrivial positive linear functional on $l^{\infty}$ with $c_{0} \subset \operatorname{ker} p$ (for example any Banach limit will do). Then $f=p \circ \phi=q \circ \tau$ for some positive linear functional $q$, so $f$ is a trace and $f(|A|)=p(1,1,1, \ldots)>0$.

Now we prove the opposite implication. Put $t_{1}=s_{1}$ and

$$
t_{n}=\frac{S_{k^{L(n)}}-S_{k^{L(n)-1}}}{k^{L(n)}-k^{L(n)-1}}
$$

where $L(n)$ is the integer defined by $k^{L(n)-1}<n \leq k^{L(n)}(n=2,3, \ldots)$. Let $D=\operatorname{diag}(t)$ with respect to some orthonormal basis. Then $s(D)=t$. Since $t_{n} \leq s_{k^{L(n)-1+1}}$ and $L(k(n-1)+1)=L(n)+1$ we have $t_{(n-1) k+1} \leq$ $s_{k L(n)+1} \leq s_{n}$ so $D \in \mathscr{I}_{k}(A)$. Quite similarly $A \in \mathscr{J}_{k}(D)$. Let $W$ be such an isometry that $W^{*}$ has an infinite dimensional kernel. Put $D^{\prime}=W D W^{*}$. Then $D=W^{*} D^{\prime} W$, so $\mathscr{I}\left(D^{\prime}\right)=\mathscr{I}(D)=\mathscr{I}(A)$. Suppose now that $A$ is regular. By Lemma 1 we can assume that the positive integer $k$ is fixed so that $\inf _{n} S_{k n} / S_{n} \geq 3$. For $j=1,2, \ldots, k$ define the linear transformations $V_{j}^{\prime}$ by $V_{j}^{\prime} e_{n}\left(D^{\prime}\right)=e_{k(n-1)+j}$ and denote a unitary extension of each $V_{j}^{\prime}$ by $V_{j}$. Put $D_{j}=V_{j} D^{\prime} V_{j}^{*}$ and $F=\sum_{j=1}^{k} D_{j}$. It is clear that $F$ is zero on the kernel of $W^{*}$ and that $F e_{n}\left(D^{\prime}\right)=u_{n} e_{n}\left(D^{\prime}\right)$ where

$$
\begin{aligned}
u_{k(n-1)+j} & =t_{n}=\frac{S_{k^{L(n)}}-S_{k^{L(n)-1}}}{k^{L(n)}-k^{L(n)-1}} \leq \frac{S_{k^{L(n)}}}{k^{L(n)}-k^{L(n)-1}} \\
& \leq \frac{1}{2} \frac{S_{k^{L(n)+1}}-S_{k^{L(n)}}}{k^{L(n)}-k^{L(n)-1}}=\frac{k}{2} t_{k(n-1)+j}
\end{aligned}
$$

$(n=1,2, \ldots, j=1,2, \ldots, k,(n, j) \neq(1,1))$. Consequently $2 F \leq k D^{\prime}$, and if $f$ is any trace on $\mathscr{I}(A)$, then

$$
2 k f\left(D^{\prime}\right)=2 \sum_{j=1}^{k} f\left(D_{j}\right)=2 f(F) \leq k f\left(D^{\prime}\right),
$$

so $f\left(D^{\prime}\right) \leq 0$. Let $B$ be any positive element of $\mathscr{I}(A)$. Then $B \in \mathscr{I}_{r}\left(D^{\prime}\right)$ with some $r$, and by Lemma 2 it is easy to construct unitary operators $U_{1}^{r}, U_{2}, \ldots$, $U_{r}$ such that $B<K \sum_{i=1}^{r} U_{i} D^{\prime} U_{i}^{*}$. So

$$
0 \leq f(B) \leq r K f\left(D^{\prime}\right) \leq 0
$$

that is, $f$ is zero on the positive part of $\mathscr{I}(A)$ and consequently on the whole $\mathscr{f}(A)$. This completes the proof of Theorem 1 .

Remark 1. If $A$ is eccentric, then (v) of Lemma 1 is satisfied by some $\left(n_{k}\right)$. We can even assume (replace $\left(n_{k}\right)$ with a subsequence if necessary) that $S_{k n_{k}} /$ $S_{(k+1) n_{k+1}} \rightarrow 0(k \rightarrow \infty)$. Then it is easy to construct a sequence of complex 
numbers $\left(\gamma_{n}\right)$ such that $\left|\gamma_{n}\right|=1$ and $\tau\left(\operatorname{diag}\left(s_{n} \gamma_{n}\right)\right)=$ an arbitrary element in the unit ball of $l^{\infty} / c_{0}$ given in advance. So $\tau$ maps $\mathscr{I}(A)$ onto $l^{\infty} / c_{0}$.

Remark 2. Condition (iii) of Lemma 1 was used in [5] for constructing unitary invariant ideals in $\mathscr{K}(\mathscr{H})$ which are not ideals in $\mathscr{B}(\mathscr{H})$. The existence of a nontrivial p.u.l. functional on $\mathscr{J}_{u}(A)$, the smallest unitary invariant ideal in $\mathscr{K}(\mathscr{H})$ containing $A$, was a side product of the construction. Now the situation is reversed, since Theorem 1 implies the theorem of [5] even without the assumption that $0 \leq A$ :

Corollary 1. If $A$ is eccentric then $\mathscr{J}_{u}(A) \neq \mathscr{I}(A)$.

Proof. If $A$ is eccentric then we can assume that $\tau$ is onto. Then for any nontrivial $q \in\left(l^{\infty} / c_{0}\right)^{*}$ with $q(\tau(A))=0$, we have that $\mathscr{I}(A) \neq \operatorname{ker} q \circ \tau \supset$ $\mathscr{J}_{u}(A)$. Indeed, for the inclusion it is enough to show that $K \mathscr{I}(A)$ and $U^{*} A U$ are contained in $\operatorname{ker} q \circ \tau$ for every compact $K$ and unitary $U$. Now $S(X)=$ $O(S(A))$ and $s(K X)=o(s(X))$ if $K$ is compact [1]; so, by an elementary argument $S(K X)=o(S(A))$ if $X \in \mathscr{I}(A)$ and consequently $\mathscr{K}(\mathscr{H}) \mathscr{I}(A)=$ $\mathscr{I}(A) \mathscr{K}(\mathscr{H})$ is contained in the kernel of $\tau$. Also, $q \circ \tau\left(U^{*} A U\right)=q \circ \tau(A)=0$. The proof is complete.

\section{NORM IDEALS}

In this section we assume that the reader is familiar with the basic results about norm ideals (we refer to chapter III of [2]). Let $c_{0}^{+}$denote the positive part of $c_{0}$. For a nonincreasing sequence $t \in c_{0}^{+}$with $t_{1}=1$, define on $\mathscr{K}(\mathscr{H})$ the functional \|\|$_{T}$ by

$$
\|X\|_{T}=\sup _{n} \frac{S_{n}(X)}{T_{n}}
$$

where $T_{n}=\sum_{i}^{n} t_{i}$ and let $\mathscr{S}_{T}=\left\{X: X \in \mathscr{K}(\mathscr{H}),\|X\|_{T}<\infty\right\} . \mathscr{S}_{T}$ is a norm ideal (called the Gohberg-Krein norm ideal) with the norm \|\|$_{T}$. We introduce the following notations:

$$
\begin{aligned}
\|\|_{A} & =\|\|_{S(A) /\|A\|}, \quad \mathscr{M}(A)=\mathscr{S}_{S(A) /\|A\|} \\
\mathscr{M}^{0}(A) & =\operatorname{cl}_{A} \mathscr{F}(\mathscr{H})=\operatorname{ker} d, \quad \mathscr{N}(A)=\operatorname{cl}_{A} \mathscr{I}(A)
\end{aligned}
$$

for an arbitrary nonzero compact operator $A$ where $\mathscr{F}(\mathscr{H})$ is the ideal of finite rank operators, $\mathrm{cl}_{A}$ is the closure operation with respect to \|\|$_{A}$ and $d(X)=\lim \sup _{n} S_{n}(X) / S_{n}(A)$ (see [2]). The following lemma entitles us to call $\mathcal{N}(A)$ the principal norm ideal generated by $A$.

Lemma 5. If $\mathcal{N}$ is any norm ideal with the norm \|\|$_{*}$, containing $A$, then $\mathscr{N} \supset \mathscr{N}(A)$.

Proof. Let $B \in \mathscr{N}(A)$. Then $S_{n}(B) \leq\|B\|_{A} S_{n}(A)$, so by a result of $\mathrm{K}$. Fan (see [2]) and by our assumptions

$$
\|B\|_{0}:=\sup _{n}\left\|B E_{n}(B)\right\|_{*} \leq\|B\|_{A} \sup _{n}\left\|A E_{n}(A)\right\|_{*}=\|B\|_{A}\|A\|_{0}
$$


where $\mathscr{N}$ is a norm ideal with the norm \|\|$_{0}$ [2] $\left(E_{n}\right.$ is as in the proof of Theorem 1). So \|\|$_{0} \leq\|A\|_{0}\|\|_{A}$ on $\mathscr{N}(A)$. Since $\mathscr{N} \supset \mathscr{I}(A)$,

$$
\mathscr{N} \supset \operatorname{cl}_{0} \mathscr{I}(A) \supset \mathrm{cl}_{A} \mathscr{I}(A)=\mathscr{N}(A)
$$

as stated.

Remark 3. If $A$ is irregular and $\|A\|=1$, then $\tau$ of Theorem 1 is bounded by \|\|$_{A}$, so it extends by continuity to a contractive p.u.l. map $\hat{\tau}$ from $\mathscr{N}(A)$ into $l^{\infty} / c_{0}$. It is proved in [2] that if $A$ is nuclear, then $\mathscr{M}^{0}(A)=\mathscr{M}(A)=$ the trace $\operatorname{class}(=\mathscr{N}(A)$ by $(4))$. Clearly $\sum s_{n}(A) \hat{\tau}=\pi(1,1, \ldots) \operatorname{tr}$. So if $A$ is irregular, then $\mathscr{N}(A)$ with $\hat{\tau}$ is a generalization of the trace class with the classical trace on it. On the other hand, if $A$ is eccentric and $\left(n_{k}\right)$ is as in Remark 1, then $\hat{\tau}$ maps the unit ball of $\mathscr{I}(A)$ onto the unit ball of $l^{\infty} / c_{0}$. Consequently $\hat{\tau}^{*}$ is a p.u.l. isometry from $\left(l^{\infty} / c_{0}\right)^{*}$ into the unitary invariant part of $\mathscr{N}(A)^{*}$. So the space of traces on $\mathscr{N}(A)$ is far from being trivial; in fact, it contains the positive part of the dual of $l^{\infty} / c_{0}$.

We leave it to the reader to prove that if $v \in \mathscr{N}(A)^{* u}$, then $\mathscr{M}^{0}(A) \subset \operatorname{ker} v$ if $A$ is not nuclear, and that $\mathscr{K}(\mathscr{H}) \mathscr{N}(A) \subset \mathscr{M}^{0}(A)$. So we have

Corollary 2. If $A$ is eccentric and $L$ is a nontrivial subset of $\mathscr{N}(A)^{* u}$ with

$$
A \in \mathscr{J}_{L}:=\bigcap_{L} \operatorname{ker} l
$$

then $\mathscr{J}_{L}$ is a unitary invariant ideal in the ring $\mathscr{K}(\mathscr{H})$. It is also closed in $\mathscr{N}(A)$, contains $A$, but does not contain $\mathscr{J}(A)$, so it is not an ideal.

Remark 4. It is clear that

$$
\mathscr{I}_{1}(A) \subset \mathscr{I}(A) \subset \mathscr{N}(A) \subset \mathscr{M}(A), \quad \mathscr{M}_{0}(A) \subset \mathscr{N}(A) .
$$

The use of regularity was realized in [2], since if $A$ is regular and $S(B)=$ $O(S(A))$ (i.e. $B \in \mathscr{M}(A))$, then

$$
s_{n}(B) \leq \frac{S_{n}(B)}{n}=O\left(\frac{S_{n}(A)}{n}\right)=O\left(s_{n}(A)\right) \quad(n \rightarrow \infty)
$$

that is $\mathscr{I}_{1}(A)=\mathscr{M}(A)$ so we simply have $\mathscr{J}(A)=\mathscr{N}(A)=\mathscr{M}(A)$. Our aim is to show that the picture becomes as diversified as can be when $A$ is eccentric.

Theorem 2. $\mathscr{N}(A)=\mathscr{M}(A)$ if and only if $A$ is not eccentric.

Proof. If $A$ is nuclear or regular, then $\mathscr{M}(A)=\mathscr{N}(A)$ by Remarks 3 and 4 . On the other hand, if $A$ is eccentric choose $\left(n_{k}\right)$ as in Remark 1. Define $g$ and $h$ by

$$
g(B)=\limsup _{k \rightarrow \infty} \frac{S_{k n_{k}}(B)}{S_{k n_{k}}(A)}, \quad h(B)=\limsup _{k \rightarrow \infty} \frac{S_{3 k n_{k}}(B)}{S_{k n_{k}}(A)}
$$

$(B \in \mathscr{M}(A))$. Clearly $g$ and $h$ are subadditive (since $S_{n}$ is subadditive, [2]) and $g \leq h \leq 3\|\|_{A}$, so they are continuous on $\mathscr{M}(A)$. Furthermore,

$$
g(B) \leq h(B) \leq g(B)+\limsup _{k \rightarrow \infty} \frac{S_{3 k n_{k}}(B)-S_{k n_{k}}(B)}{S_{n_{k}}(A)}=g(B)
$$


if $B \in \mathscr{I}(A)$ (see the proof of Lemma 4). So $\mathscr{I}(A)$, and consequently $\mathscr{N}(A)$, are contained in the kernel of the continuous functional $h-g$. Now, for any nondecreasing concave sequence $Z$ of positive numbers with $Z_{n}=o(n)$ there is an operator $B_{Z}$ such that $S\left(B_{Z}\right)=Z$. So let $Z_{3 k n_{k}}=S_{3 k n_{k}}(A)$ and let $Z$ be linear on $\left[3 k n_{k}, 3(k+1) n_{k+1}\right](k=1,2, \ldots)$. Then $h\left(B_{Z}\right)=1$, and an elementary computation shows (observing that necessarily $k n_{k} /(k+1) n_{k+1} \rightarrow 0$ ) that

$$
g\left(B_{Z}\right)=\limsup _{k \rightarrow \infty} \frac{Z_{k n_{k}}}{S_{n_{k}}(A)}=\frac{1}{3}
$$

Consequently $B_{Z}$ is not in $\mathscr{N}(A)$, but it clearly is in $\mathscr{M}(A)$. The proof is complete.

Remark 5. If $A$ is not nuclear, then $A \notin \mathscr{M}^{0}(A)$ [2]; so if $A$ is even eccentric, we have $\mathscr{M}^{0}(A) \neq \mathscr{N}(A) \neq \mathscr{M}(A)$ by Theorem 2 . The discovery of such intermediate norm ideals is due to B. S. Mityagin [3]. The above proof gives a new way of constructing (perhaps new types of) intermediate norm ideals.

Remark 6. If $A$ and $\left(n_{k}\right)$ are as in the above proof, then $\pi \circ \phi \mid \mathscr{M}(A)$ is not linear. To help on this, choose $\left(n_{k}\right)$ so that even $S_{3^{k} n_{k}}(A) / S_{n_{k}}(A) \rightarrow 1$. For a double sequence of projections $P=\left(P_{k}^{(j)}\right)$ put $\Phi_{P}=k^{-1} \sum_{j=0}^{k-1} \phi_{P^{(j)}}$. Let $P_{k}^{(j)}(B)=E_{3 j n_{k}}(B)$ and define $\Phi$ accordingly. As before, we can prove that $\mathscr{T}=\pi \circ \Phi \mid \mathscr{M}(A)$ is a p.u.l. map and that it has the same properties as $\hat{\tau}$. Indeed, if $B \in \mathscr{M}(A)$ and the double sequence $R$ is tied to $P(B)$, then

$$
\begin{aligned}
S_{n_{k}}(A)\left|\left(\Phi_{R}-\Phi\right)(B)(k)\right| \leq k^{-1} \sum_{j=0}^{k-1}\left(S_{3 \cdot 3 j n_{k}}(B)-S_{3 j n_{k}}(B)\right) \\
=k^{-1}\left(S_{3^{k} n_{k}}(B)-S_{n_{k}}(B)\right) \leq k^{-1}\|B\|_{A} S_{3^{k} n_{k}}(A)=o\left(S_{n_{k}}(A)\right)
\end{aligned}
$$

Theorem 3. $\mathscr{I}(A)=\mathscr{N}(A)$ if and only if $A$ is regular.

Lemma 6. Let $s$ be a nonincreasing irregular sequence in $c_{0}^{+} \backslash\{0\}$. Then there exists a nonincreasing sequence $t$ such that $T=O(S) \quad\left(T_{n}=\sum_{1}^{n} t_{i}\right)$ but $t \neq$ $O(s)$. Moreover, if $\sum s_{n}=\infty$, then $t$ can be chosen so that $T=o(S)$.

Proof. Let $\left(n_{k}\right)$ be such an increasing sequence for which $S_{n_{k}}>3^{k} n_{k} s_{n_{k}}$ and for which $S_{n_{k}}>2 S_{n_{k-1}}$ in case $\sum s_{n}=\infty \quad(k=2,3, \ldots)$. Put $T_{n_{k}}=S_{n_{k}}(1-$ $2^{-k}$ ) and extend $T$ linearly between neighboring $n_{k}$ 's. If $\sum S_{n}=r<\infty$, we have that $s_{n_{k}}<r 3^{-k} / n_{k}$ and $t_{n_{k}} \geq 2^{-k} S_{n_{k-1}} / n_{k} \geq s_{1} / 2^{k} n_{k}$. If $\sum s_{n}=\infty$ then

$$
t_{n_{k}} \geq\left(1-2^{-k}\right) \frac{S_{n_{k}}-S_{n_{k-1}}}{n_{k}} \geq \frac{1}{2 n_{k}} \frac{S_{n_{k}}}{2} \text {. }
$$

In any case $t \neq O(s)$, but clearly $T=O(S)$. For the second statement we note that if $\left(\varepsilon_{n}\right)$ decreases to zero slowly enough, then still $t_{n}^{\prime}=\varepsilon_{n} t_{n} \neq O\left(s_{n}\right)$ but $T^{\prime}=o(T)=o(S)$ by an elementary argument. 
Proof of Theorem 3. One way is clear by Remark 4. If $A$ is irregular, then by Lemma 6 there is an $X \in \mathscr{K}\left(\mathscr{H}^{\prime}\right)$ such that $X \oplus 0 \oplus 0 \oplus \cdots$ is in $\mathscr{M}^{0}(A)$ but not in $\mathscr{I}_{1}(A) \quad\left(\mathscr{H}=\bigoplus_{1}^{\infty} \mathscr{H}^{\prime}\right)$. Then by Lemma $2, X \oplus \cdots \oplus X \oplus 0 \oplus \cdots \notin \mathscr{I}_{n}(A)$ ( $n$ nonzero terms). Put $Y=\oplus_{1}^{\infty} 2^{-n} X$. The sum clearly converges in \|\|$_{A}$ so $Y \in \mathscr{M}^{0}(A) \subset \mathscr{N}(A)$. But obviously $Y \notin \mathscr{I}_{n}(A) \quad(n=1,2, \ldots)$, so $Y \notin \mathscr{I}(A)$.

Proof of Theorem IRR. The equivalence of (a), (b), (c), and (f) follows by Theorem 1 and by Remarks 3, 4, and 6. Suppose $\mathscr{I}(A)=\mathscr{N}(B)$. Then $\mathscr{I}(A)=\mathscr{N}(A)$ by Lemma 5 , so $A$ is regular by Theorem 3 . By the above equivalence, $B$ is regular too, so (f) implies (d) and (e). The converse statements are clear by Remark 4 .

Proof of Theorem ECC. By Remarks 4 and 5, (g) is equivalent to (k). Also (i) and $(\mathrm{j})$ are equivalent to $(\mathrm{k})$ by Theorem 1 and by Remarks 3, 4, and 6 . Suppose $\mathscr{M}(A)=\mathscr{N}(B)$. By the maximality of $\mathscr{M}(A)$ [2], $\mathscr{N}(B)=\mathscr{M}(B)$; so $B$ is not eccentric by Theorem 2. By the equivalence of $(\mathrm{j})$ and $(\mathrm{k}), A$ is not eccentric, so $(\mathrm{k})$ implies $(\mathrm{h})$. The converse is clear by Theorem 2 .

\section{Questions:}

(1) Is $\mathscr{J}_{u}(A)=\mathscr{I}(A)$ if $A$ is not eccentric?

(2) Does it follow that $\mathscr{N}(A)^{* u}=\left(l^{\infty} / c_{0}\right)^{*}$ if $A$ is eccentric?

(3) Is

$$
[\mathcal{N}, \mathscr{B}(\mathscr{H})]=\bigcap_{\mathscr{N} * u} \operatorname{ker} l
$$

if $\mathscr{N}=\mathscr{N}(A)$ or $\mathscr{N}=\mathscr{M}(A)$ and $A$ is not nuclear?

\section{REFERENCES}

1. C. K. Fong and H. Radjavi, On ideals and Lie ideals of compact operators, Math. Ann. B 262 (1963), 23-28.

2. I. C. Gohberg and M. G. Krein, Introduction to the theory of nonselfadjoint operators, Trans. Amer. Math. Soc., Vol. 18, Providence, R.I., 1969.

3. B. S. Mitjagin, Normed ideals of intermediate type, Izv. Akad. Nauk SSSR Ser. Mat. 28 (1964), 819-832; English transl., Amer. Math. Soc. Transl. (2) 63 (1967), 180-194.

4. N. Salinas, Symmetric norm ideals and relative conjugate ideals, Trans. Amer. Math. Soc. $\mathbf{1 8 8}$ (1974), 213-240.

5. J. V. Varga, On unitary invariant ideals in the algebra of compact operators (to appear).

Bolyai Institute, Szeged, Aradi Vértanuk Tere 1, 6720, Hungary 